\title{
bSSFP phase correction and its use in magnetic resonance electrical properties tomography
}

\author{
Safa Ozdemir | Yusuf Ziya Ider \\ Department of Electrical and Electronics Engineering, Bilkent University, Ankara, Turkey
}

\section{Correspondence}

Yusuf Ziya Ider, Department of Electrical and Electronics Engineering, Bilkent University, Ankara, Turkey.

Email: ider@ee.bilkent.edu.tr

\section{Funding information}

This study was supported by TUBITAK 114E522 research grant. Experimental data were acquired using the facilities of UMRAM, Bilkent University, Ankara.
Purpose: Balanced steady-state free precession (bSSFP) sequence is widely used because of its high SNR and high speed. However, bSSFP images suffer from "banding artifact" caused by $\mathrm{B}_{0}$ inhomogeneity. In this article, we propose a method to remove this artifact in bSSFP phase images and investigate the usage of the corrected phase images in phase-based magnetic resonance electrical properties tomography (MREPT).

Theory and Methods: Two bSSFP phase images, obtained with different excitation frequencies, are collaged to get rid of the regions containing banding artifacts. Phase of the collaged bSSFP image is the sum of the transceive phase of the RF system and an error term that depends on $\mathrm{B}_{0}$ and $\mathrm{T}_{2}$. By using $\mathrm{B}_{0}$ and $\mathrm{T}_{2}$ maps, this error is eliminated from bSSFP phase images by using pixel-wise corrections. Conductivity maps are obtained from the uncorrected and the corrected phase images using the phasebased cr-MREPT method.

Results: Phantom and human experiment results of the proposed method are illustrated for both phase images and conductivity maps. It is shown that uncorrected phase images yield unacceptable conductivity images. When only $\mathrm{B}_{0}$ information is used for phase correction conductivity, reconstructions are substantially improved, and yet $\mathrm{T}_{2}$ information is still needed to fully recover accurate and undistorted conductivity images.

Conclusions: With the proposed technique, $\mathrm{B}_{0}$ sensitivity of the bSSFP phase images can be removed by using $\mathrm{B}_{0}$ and $\mathrm{T}_{2}$ maps. It is also shown that corrected bSSFP phase images are of sufficient quality to be used in conductivity imaging.

\section{K E Y W O R D S}

banding artifact, bSSFP, conductivity, MREPT, phase-based

\section{$1 \mid$ INTRODUCTION}

Impedance imaging aims at reconstructing conductivity, $\sigma$, and permittivity, $\epsilon$, of the tissues. Earlier methods of impedance imaging are electrical impedance tomography (EIT) ${ }^{1,2}$ and magnetic induction tomography (MIT) ${ }^{3}$ that are used to induce currents in the object by using either surface electrodes (EIT) or external coils (MIT). However, these methods yield images that have low spatial resolution in interior regions because the measurements (i.e., surface potentials) are not very sensitive to electrical property perturbations relatively far from the surface. To overcome this weakness, magnetic resonance electrical impedance tomography (MREIT) has been introduced. ${ }^{4-9}$ In MREIT, current is induced by using surface electrodes in the frequency range of $10 \mathrm{~Hz}-10 \mathrm{kHz}$ and the resulting magnetic field is measured by MRI to reconstruct 
the image of electrical properties. Still, reducing the external applied current to below safety limits while having sufficient resolution is a problem in MREIT. A more recent method, magnetic resonance electrical properties tomography (MREPT), attempts to image electrical properties by measuring the magnetic field of currents induced at the Larmor frequency of the MRI system. MREPT was first suggested, in concept, by Haacke et al. in $1991,{ }^{10}$ was successfully applied for the first time by Wen in 2003, ${ }^{11}$ and systematic research on MREPT is started by Katscher et al. in 2009. ${ }^{12}$

From Maxwell's equations, the relationship between the clockwise (left-handed) rotating component of the transmit RF magnetic flux density, $\mathrm{B}_{1}^{+}$, and admittivity of the object $(\gamma=\sigma+i \omega \epsilon)$ can be derived as follows ${ }^{13}$ :

$$
-\nabla^{2} \mathrm{~B}_{1}^{+}=\frac{\nabla \gamma}{\gamma} \times\left(\nabla \times \mathrm{B}_{1}^{+}\right)-\mathrm{i} \omega \mu_{0} \gamma \mathrm{B}_{1}^{+} .
$$

With the assumption of locally constant electrical properties, known as "local homogeneity assumption" (LHA), the gradient term $\left(\frac{\nabla \gamma}{\gamma} \times\left(\nabla \times \mathrm{B}_{1}^{+}\right)\right)$is eliminated and admittivity becomes:

$$
\gamma=\frac{\nabla^{2} B_{1}^{+}}{i \omega \mu_{0} B_{1}^{+}} .
$$

Although Equation (2) is widely used for obtaining electrical property images, this method suffers greatly from boundary artifacts, because of the elimination of the gradient term in Equation (1). To solve this issue, as also reviewed in Liu et al. ${ }^{13}$ and Katscher and van der Berg, ${ }^{14}$ several approaches are followed. ${ }^{15-17}$ The approach of Hafalir et al. ${ }^{15}$ introduces the convection-reaction partial differential equation (PDE)-based method (cr-MREPT), which is, in fact, the solution of Equation (1) using numerical techniques. The approach of Liu et al. ${ }^{16}$ uses similar formulation in their method called gradient-based electrical properties tomography (gEPT). In this method, with the knowledge of $\mathrm{B}_{1}^{+}$that is obtained by using a multi-channel transceiver RF coil, gradient of the electrical properties is obtained. Through spatial integration starting from a seed-point, both conductivity and permittivity are obtained. Contrast source inversion-based EPT (CSI-EPT) views the object as a scatterer placed in the field generated by the RF coil. ${ }^{17}$ EPs are updated until the measured RF field matches closely the field calculated by the solution of the integral-equation-based forward problem expressing the magnetic field as a function of the EPs.

For the aforementioned methods, both the phase and magnitude of $\mathrm{B}_{1}^{+}$are to be measured. Although there are well-established techniques to obtain the magnitude of $\mathrm{B}_{1}^{+18-20}$ phase of $\mathrm{B}_{1}^{+}$cannot be measured directly in MRI. For birdcage-like quadrature volume coil configurations, transmit phase is roughly taken as half of the transceive phase.
This widely used approach is called transceive phase approximation (TPA). ${ }^{13,21}$ Lately, phase-based methods, which are solely based on the phase of the $\mathrm{B}_{1}^{+}$rather than the complex $\mathrm{B}_{1}^{+}$map, have emerged. ${ }^{22,23}$ Because of the difficulties in obtaining high SNR for the $\mathrm{B}_{1}^{+}$magnitude maps in a reasonable time, phase-based methods offer an advantage especially for practical clinical applications. These methods are inherently free from TPA and do not require $\mathrm{B}_{1}^{+}$magnitude mapping. ${ }^{21}$ Standard phase-based approach uses the equation:

$$
\sigma(\mathrm{x}, \mathrm{y}) \approx \frac{\nabla^{2} \phi(\mathrm{x}, \mathrm{y})}{2 \mu_{0} \omega},
$$

to find conductivity where $\phi$ is the transceive phase (if transmit phase is used, the factor 2 in the denominator is omitted). ${ }^{21}$ Although this "standard" phase-based formulation is free from TPA and does not require $\mathrm{B}_{1}^{+}$mapping, it assumes low $\mathrm{B}_{1}^{+}$magnitude gradients, in addition to using LHA. While LHA causes well-known tissue-boundary artifacts in the conductivity images as mentioned before, assuming low $\mathrm{B}_{1}^{+}$ magnitude gradient causes errors in the reconstructed conductivity values (up to $10 \%$ for $3 \mathrm{~T}$ systems) ${ }^{21}$ especially toward the boundary of the imaged object. ${ }^{24}$ Recently, a phase-based EPT method that does not require LHA, but still assumes low $\mathrm{B}_{1}^{+}$gradients, was introduced by Gurler and Ider. ${ }^{25}$ With this approach, called "phase-based cr-MREPT technique," boundary artifacts in the conductivity images are significantly reduced. (Note that the methods mentioned in the previous paragraph in reference to Hafalir et al., ${ }^{15}$ Liu et al., ${ }^{16}$ and Balidemaj et al. ${ }^{17}$ aim at reducing boundary artifacts in full $\mathrm{B}_{1}^{+}$-based methods that use both the phase and the magnitude of $\mathrm{B}_{1}^{+}$. Gurler's approach, on the other hand, is similar in concept to Hafalir et al. ${ }^{15}$ but uses only the phase of $\mathrm{B}_{1}^{+}$).

To reconstruct conductivity using the phase-based approach, any MR sequence that provides $\mathrm{B}_{1}^{+}$phase can be used. Commonly used sequences are spin-echo, ${ }^{23}$ gradient echo, ${ }^{26} \mathrm{UTE} / \mathrm{ZTE},{ }^{27,28}$ and balanced steady-state free precession (bSSFP). ${ }^{29}$ Spin-echo suffers from eddy current effects and lengthy acquisition time. Gradient echo suffers from offresonance effect, which needs to be corrected by additional $\mathrm{B}_{0}$ mapping. In addition to this, eddy currents and acquisition time are still problematic. UTE/ZTE, despite being fast sequences, suffer from streaking artifacts that deteriorate the phase images, and such artifacts can be amplified through Laplacian operation and distort the final conductivity image. bSSFP is favorable to obtain phase images because it has high speed, high SNR, motion insensitivity, and automatic eddy current compensation because of balanced gradients. ${ }^{14,29}$ On the other hand, it suffers greatly from $\mathrm{B}_{0}$ inhomogeneity and the concomitant "banding artifact." In regions of banding artifact, MR signal reduces significantly and also phase errors occur. As shown later, for low $T_{2}$ values, these phase errors are even more prominent. 
The purpose of this study is to correct the phase errors in images obtained by the bSSFP sequence to make it an acceptable sequence for phase-based MREPT. In general, a method that makes use of $B_{0}$ and $T_{2}$ maps, and 2 bSSFP runs with an excitation frequency difference of $(2 \mathrm{TR})^{-1}$ is developed to correct the phase distribution obtained by bSSFP. Following the sections that explain the theory and the rational of the method, phantom image reconstructions and head data results using phase-based cr-MREPT technique are presented.

\section{\begin{tabular}{l|l}
2 & THEORY
\end{tabular}}

\section{1 | Dependence of bSSFP phase on $\Delta \mathbf{B}_{0}$ and $T_{2}$}

bSSFP sequence using alternating RF pulses is shown in Figure 1. In bSSFP imaging, the complex image value at an arbitrary pixel in the slice of interest, can be expressed as ${ }^{30,31}$ :

$S=K M=K M_{1} e^{-T E / T_{2}} \mathrm{e}^{\mathrm{i} 2 \pi\left(\mathrm{f}_{\mathrm{OR}}+\Delta \mathrm{f}_{\mathrm{s}}\right) \mathrm{TE}} \frac{1+a \mathrm{e}^{-i 2 \pi\left(\mathrm{f}_{\mathrm{OR}}+\Delta f_{s}\right) T R}}{1+b \cos \left(2 \pi\left(\mathrm{f}_{\mathrm{OR}}+\Delta \mathrm{f}_{s}\right) T R\right)}$,

where.

$$
\begin{gathered}
M_{1}=M_{0} \frac{\left(1-E_{1}\right) \sin \alpha}{1-E_{1} \cos \alpha-\left(E_{1}-\cos \alpha\right) E_{2}^{2}}, \\
a=E_{2}, \\
b=E_{2} \frac{1-E_{1}-E_{1} \cos \alpha+\cos \alpha}{1-E_{1} \cos \alpha-\left(E_{1}-\cos \alpha\right) E_{2}^{2}},
\end{gathered}
$$
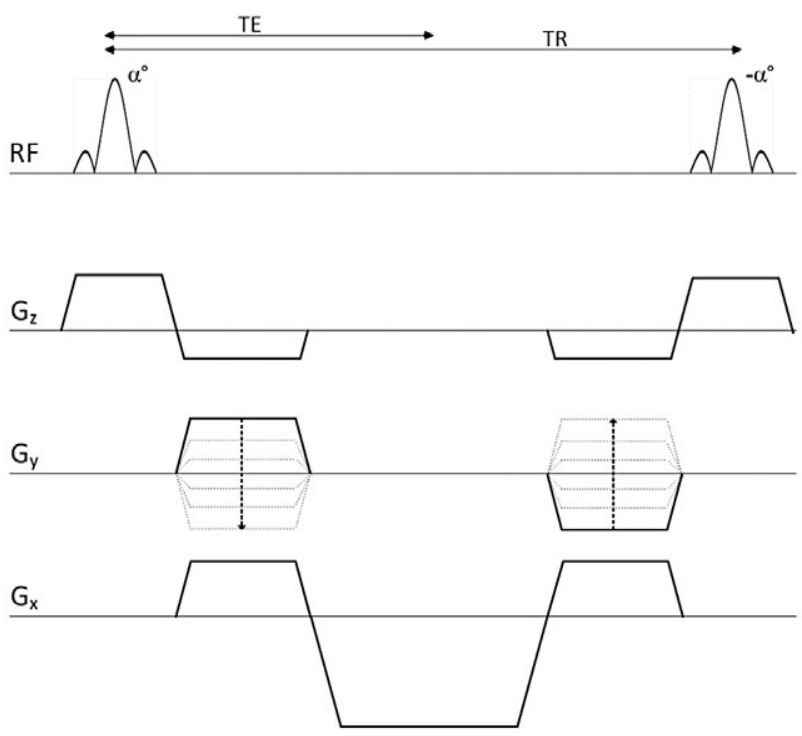

$A D C$

F I G URE 1 Pulse sequence diagram for the RF alternating balanced steady-state free precession (bSSFP) sequence used in this study

$$
\begin{aligned}
& E_{1}=e^{-\frac{T R}{T_{1}}}, \\
& E_{2}=e^{-\frac{T R}{T_{2}}} .
\end{aligned}
$$

$\mathrm{T}_{1}$ and $\mathrm{T}_{2}$ represent longitudinal and transversal relaxation times, TE and TR are echo time and repetition time, $\alpha$ is flip angle, $\mathrm{M}_{0}$ is equilibrium magnetization, $f_{\mathrm{OR}}$ is off-resonance frequency because of $\Delta \mathrm{B}_{0}, \Delta \mathrm{f}_{\mathrm{s}}$ is the frequency shift in the excitation frequency as controlled by the user, and $K$ is the complex valued factor the phase of which is the transceive phase of the RF system (sum of transmit phase and receive phase). Although magnitude of $S$ is dependent on many parameters, phase of it, which is the measured bSSFP phase, is relatively simple and can be expressed as:

$$
\angle(S)=\angle(K)+\angle(M),
$$

where

$$
\Delta(M)=2 \pi\left(\mathrm{f}_{\mathrm{OR}}+\Delta \mathrm{f}_{s}\right) T E+\Delta\left(1+E_{2} e^{-i 2 \pi\left(\mathrm{f}_{\mathrm{OR}}+\Delta \mathrm{f}_{s}\right) T R}\right)
$$

In Equation (10), $\angle(K)$ is what we are interested in and $\angle(M)$ can be viewed as a phase error term. In the expression for the phase error term, the independent variables are $\mathrm{T}_{2}$ (through $\mathrm{E}_{2}$ ), the off-resonance frequency $f_{\mathrm{OR}}, \mathrm{TE}, \mathrm{TR}$, and the excitation frequency shift $\Delta f_{s}$. Among these, TE, TR, and the excitation frequency shift are determined by the user. Therefore, by making use of methods for determining the offresonance frequency and $\mathrm{T}_{2}$ values for each pixel, we can obtain the correct value of transceive phase for each pixel using Equations (10) and (11). Note that, if $\mathrm{E}_{2}=1$ (infinite $\mathrm{T}_{2}$ ), then $\angle(M)$ is either 0 or $\pi$ (see Appendix).

Figure 2 demonstrates magnitude and phase of $M$ with respect to off-resonance as obtained from Equation (4). Figure $2 \mathrm{~A}$ and $\mathrm{B}$ are for a large $\mathrm{T}_{2}\left(\mathrm{~T}_{2}=1000 \mathrm{~ms}\right)$, and the graphs are given for two cases: (1) frequency shift $\Delta f_{s}$ equals 0 (blue lines), and (2) frequency shift $\Delta f_{s}$ equals $1 / 2 \mathrm{TR}$ (red lines). Concentrating on the magnitude graph for 0 frequency shift (the blue line in Figure 2A), we see that, for 0 off-resonance, the magnitude of $M$ is maximum, and as off-resonance increases, the magnitude begins to decrease and the decrease becomes substantial as the off-resonance approaches $(2 \mathrm{TR})^{-1}$ (banding artifact). The magnitude increases again with further increase in off-resonance, and this off-resonance dependent behavior is periodic with a period of $(\mathrm{TR})^{-1}$. The off-resonance frequency range is divided into 2 parts, called the "pass-band" and the "stop-band" regions. ${ }^{32}$ The passband region corresponds to a region in which magnitude is high, and stop-band region corresponds to the region where magnitude is low. These 2 regions are non-intersecting and their union covers the whole range. The exact definitions of these regions depend on the application and the purpose of 


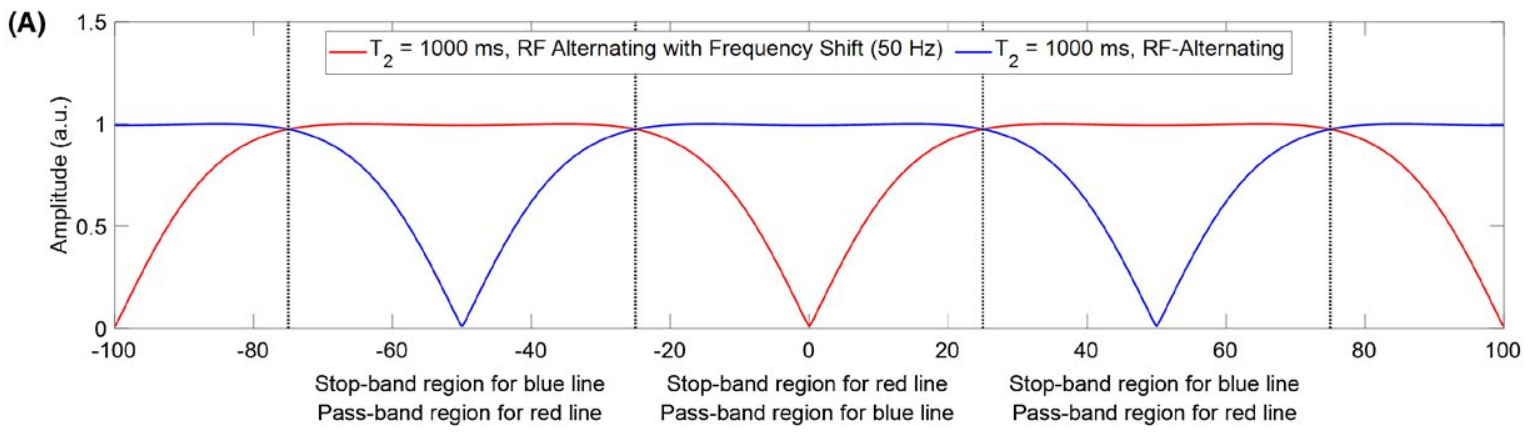

(B)

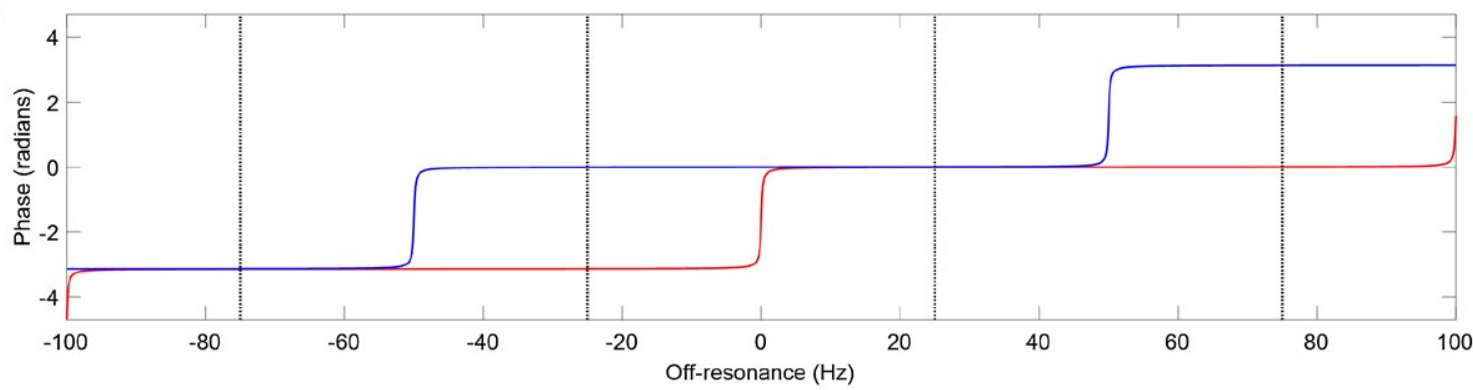

(C)

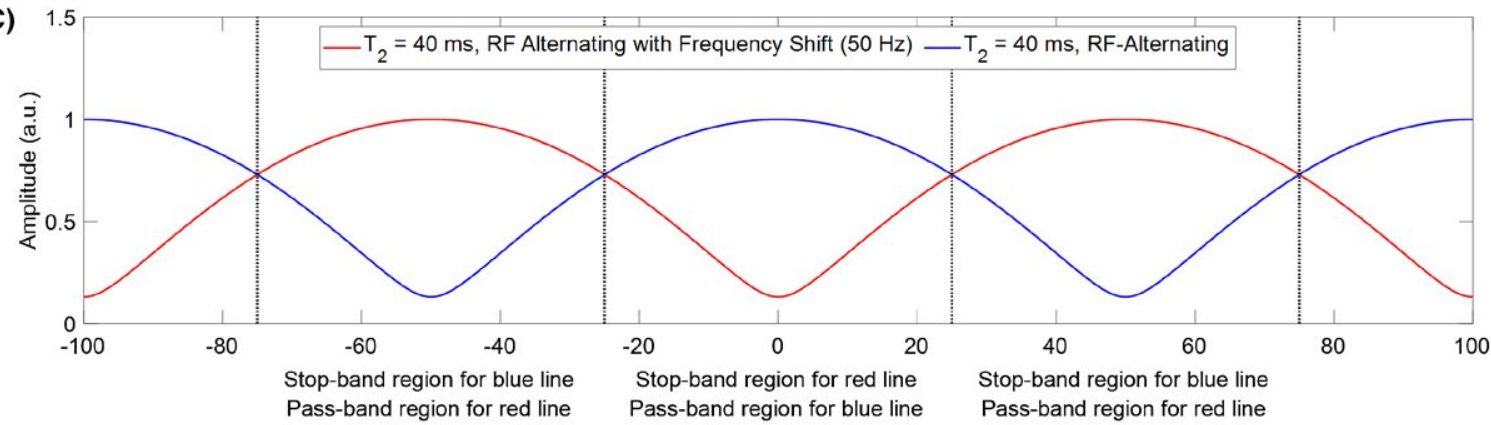

(D)

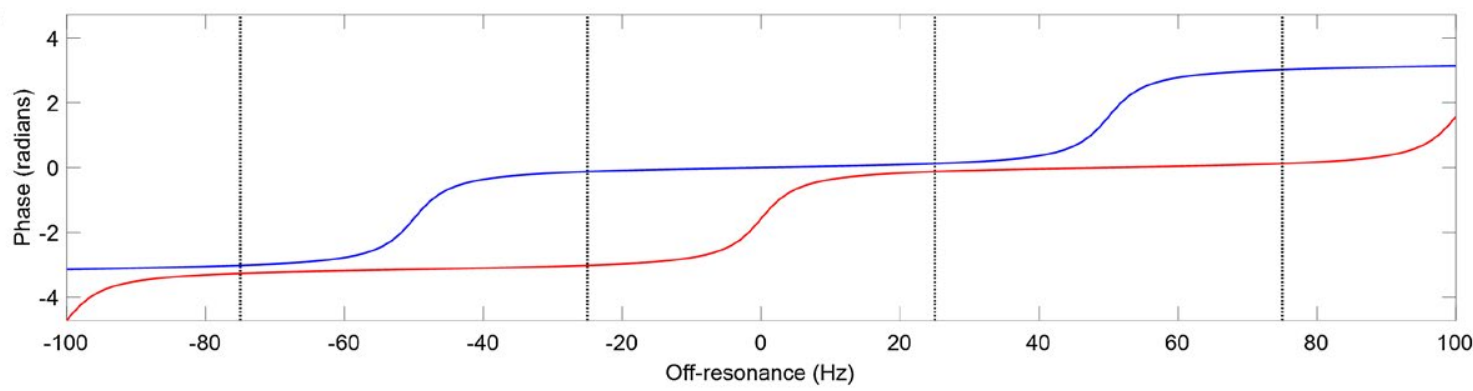

F I G URE 2 (A) Magnitude and (B) phase of the steady-state magnetizations as a function of off-resonance for $\mathrm{T}_{2}=1000 \mathrm{~ms}$, and (C and D) $\mathrm{T}_{2}=40 \mathrm{~ms}$ are shown. Blue lines correspond to the magnetizations that are obtained with bSSFP sequence using alternating RF pulse, whereas red lines correspond to the same sequence but excitation frequency difference of $50 \mathrm{~Hz}$. Simulation parameters are: $\mathrm{T}_{1}=6000 \mathrm{~ms}$, TE $=5 \mathrm{~ms}$, $\mathrm{TR}=10 \mathrm{~ms}$, flip angle $=40^{\circ}$

the study. ${ }^{32}$ In our case, we have decided to determine them as follows: in the base period $\left[-\frac{1}{2 \mathrm{TR}}<f<\frac{1}{2 \mathrm{TR}}\right]$, pass-band is defined as $\left[-\frac{1}{4 \mathrm{TR}}<f<\frac{1}{4 \mathrm{TR}}\right]$, and the stop-band is the rest of the period. The boundaries of these regions are shown in Figure 2 for the base period and also for the adjacent periods. For the graph for which excitation frequency shift is equal to $(2 \mathrm{TR})^{-1}$ (the red lines in Figure 2A and B), we observe that the magnitude and phase graphs shift exactly by an amount of $(2 \mathrm{TR})^{-1}$ with respect to the blue lines. Therefore, using the same definition of pass-band and stop-band regions for these red graphs reveal regions such that the pass-band region of the blue graphs corresponds exactly to the stop-band region of the red graph and vice versa. The reason why stop-band and pass-band regions are selected as explained above will be apparent later.

When we look at the phase graphs, we observe that in the pass-band regions, phases are almost constant; however, in the 
middle of stop-band regions, a jump of $\pi$ radians is observed. This jump corresponds to the phase jump in the banding artifact of bSSFP images. When the blue phase lines and the red phase lines are compared, it is observed that the difference of the phase in the pass-band region of the red line and the phase in the pass-band region of the blue line may be either 0 or $\pi$ radians except for a very narrow transition region. Please note that these observations are made for the case of large $T_{2}$ (i.e., $\mathrm{T}_{2}=1000 \mathrm{~ms}$ ). For infinite $\mathrm{T}_{2}$, the aforementioned narrow transition region would be infinitesimally small.

Figure $2 \mathrm{C}$ and $\mathrm{D}$ present the graphs for a small $\mathrm{T}_{2}\left(\mathrm{~T}_{2}\right.$ $=40 \mathrm{~ms}$ ). One can observe that the magnitude plots do not display a constant plateau behavior, even in the pass-band region, and begin to decrease at lower off-resonance frequencies. Similarly, the phase graphs also do not display almost constant behavior in the pass-band regions but display a variation dependent on both $\Delta \mathrm{B}_{0}$ and $\mathrm{T}_{2}$. For example, for small $\Delta \mathrm{B}_{0}$, dependence on $\Delta \mathrm{B}_{0}$ is almost linear, and the slope is equal to $\frac{1}{2}-\frac{E_{2}}{1+E_{2}}$. We called this variation of phase in the pass-band regions "non-constant plateau effect."

\section{3 | METHODS}

\section{1 | Method for correcting phase errors because of $\Delta B_{0}$ and $T_{2}$}

The method comprises the acquisition of 2 bSSFP images, 1 $\mathrm{B}_{0}$ map, and $1 \mathrm{~T}_{2}$ map. Using the information contained in the $\mathrm{B}_{0}$ map, a bSSFP image is segmented into 2 segments (set of pixels). One segment corresponds to pixels for which $\Delta \mathrm{B}_{0}$ lies in the pass-band region of the magnetization versus off-resonance curves shown in Figure 2, and the other segment contains pixels at which $\Delta \mathrm{B}_{0}$ corresponds to the stop-band region.

The 2 bSSFP images are obtained with different excitation frequencies. Difference between the 2 excitation frequencies is selected as $(2 \mathrm{TR})^{-1}$, because periodicity of regions (pass-band and stop-band) is (TR $)^{-1}$ (how these excitation frequencies are determined is explained in Sequence Protocols section). In this way, we have complementary images such that, if 1 pixel lies in the pass-band segment in 1 bSSFP image, it lies in the stop-band segment in the other bSSFP image. The boundaries between the pass-band and stop-band segments are called "the band transition boundaries." A third image is generated by combining the pass-band segments extracted from each of the bSSFP images. In other words, this "collaged" image is formed by making use of the banding artifact-free segments of each of the 2 bSSFP images. However, the phase of the collaged image still has contributions from the phase error term explained in Equations (10) and (11). Phase error is calculated for any pixel by $\angle(M)$ as given in Equation (11), using the $\mathrm{T}_{2}$ and $\Delta \mathrm{B}_{0}$ values of that pixel. Calculated phase error is subtracted from the collaged image to obtain $\Delta \mathrm{B}_{0}$ and $\mathrm{T}_{2}$ corrected phase maps. If $\mathrm{T}_{2}$ map is not available, one can take
$\mathrm{E}_{2}=1$ and make the phase correction accordingly, in which case the corrected phase image is called "type-I corrected phase image." If both $\Delta \mathrm{B}_{0}$ and $\mathrm{T}_{2}$ maps are available, $\mathrm{E}_{2}$ is assigned to its correct value for each pixel and the corrected phase image is called "type-II corrected phase image."

\section{2 | Phase based cr-MREPT}

Instead of the standard phase-based MREPT method expressed by Equation (3), the approach by Gurler and Ider is used to obtain conductivity from the phase maps. ${ }^{25}$ This method is based on the convection-reaction PDE for resistivity, $\rho$, as given below:

$$
(\nabla \varphi \cdot \nabla \rho)+\left(\nabla^{2} \varphi\right) \rho-2 \omega \mu_{0}=0,
$$

where $\rho=1 / \sigma$, and $\phi$ is the transceive phase. The convection term $(\nabla \varphi \times \nabla \rho)$ helps to combat the boundary artifact problem. For regularization purposes, and also to minimize spurious oscillations occurring along the internal tissue boundaries, an artificial diffusion term is added to this equation as follows ${ }^{25,33}$ :

$$
-c \nabla^{2} \rho+(\nabla \varphi \cdot \nabla \rho)+\left(\nabla^{2} \varphi\right) \rho-2 \omega \mu_{0}=0,
$$

where $c$ is the constant diffusion coefficient. Because the diffusion term also has a low-pass filter effect, higher values of $c$ result in loss of resolution, whereas lower values of $c$ are not sufficient to eliminate oscillations. To find optimum value of $c$, different values from 0.001 to 0.05 are tried, and 0.015 is selected by visual inspection.

This method can be applied to any 3D region of interest (ROI). Given the 3D phase map, a 2D ROI is selected visually from any z-slice and the ROI is extended to a 3D volume ROI by specifying the number of slices to be included above and below the initial slice. For z-independent $\rho$ distributions, such as in the experimental phantom explained below, the PDE can be reduced to its 2D form and solved in a 2D ROI in the slice of interest. ${ }^{25}$ The pixels lying on the boundary of the ROI are used to specify a Dirichlet boundary condition of $\rho=0.5 \mathrm{~S} / \mathrm{m}$. It is known that the use of artificial diffusion, as shown in Equation (13), makes the solution not so sensitive to the boundary condition for voxels more than few layers inside the ROI. This issue, the numerical solution method, and the various assumptions inherent in the phase-based cr-MREPT method are explained in Gurler and Ider. ${ }^{25}$

\section{3 | Phantom setup}

A cylindrical, z-independent experimental phantom with diameter of $20 \mathrm{~cm}$ and height of $25 \mathrm{~cm}$ was constructed. For background region, agar-saline gel $(20 \mathrm{~g} / \mathrm{L}$ agar, $2 \mathrm{~g} / \mathrm{L} \mathrm{NaCl}$, 

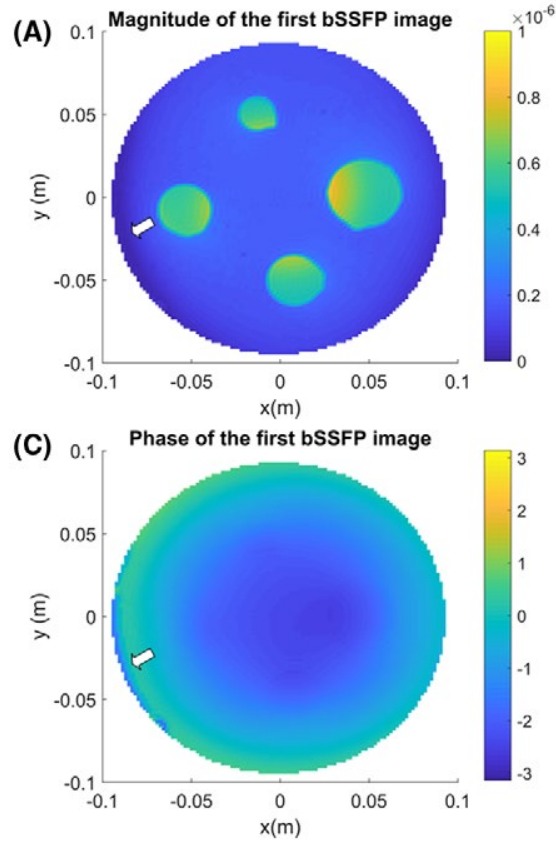

(B)

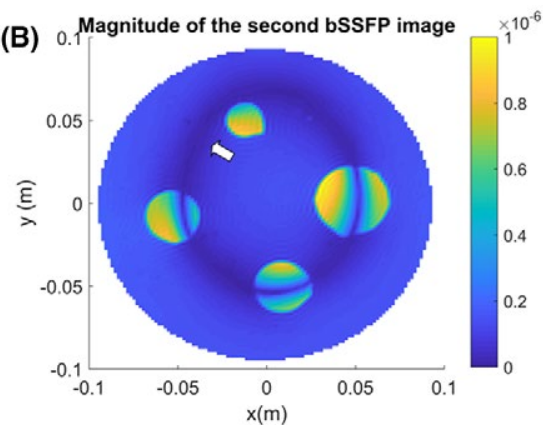

(D)

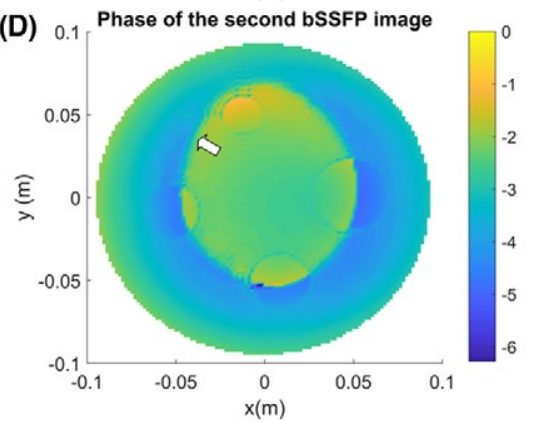

FIGURE 3 For the phantom experiment, (A and B) magnitude and $(\mathrm{C}$ and $\mathrm{D}$ ) phase images obtained by the 2 bSSFP sequences. In all images banding artifacts shown by white arrows
$0.2 \mathrm{~g} / \mathrm{L} \mathrm{CuSO}_{4}$ ) was used, and for anomalies, longitudinal holes were drilled and filled with saline solution $(6 \mathrm{~g} / \mathrm{L} \mathrm{NaCl}$, $0.2 \mathrm{~g} / \mathrm{L} \mathrm{CuSO}_{4}$ ). The conductivity values for background and the anomaly regions are expected to be 0.5 and $1 \mathrm{~S} / \mathrm{m}$, respectively. ${ }^{15}$ Anomaly regions have diameters of $0.5,1.5$, and $2.5 \mathrm{~cm}$, and they are visible in the MR magnitude image of the middle slice of the phantom in Figure 3A.

\section{4 | In vivo human experiment}

MRI scans were performed on a healthy, 25-year-old male volunteer after obtaining written informed consent in line with the approval of the Institutional Review Board of Bilkent University.

\section{5 | Sequence protocols}

For obtaining the $\mathrm{B}_{1}^{+}$phase, 2 bSSFP sequences are used (Figure 1). The sequence parameters in the phantom experiment were: $\mathrm{TE} / \mathrm{TR}=5 / 10 \mathrm{~ms}, \mathrm{FOV}=225 \mathrm{~mm}$, voxel size $=1.76 \times 1.76 \times 1.5 \mathrm{~mm}, \mathrm{FA}=40^{\circ}$, NEX (number of averages) $=32$, number of slices $=20$, and duration for each scan $=5: 29 \mathrm{~min} / \mathrm{s}$. In the human experiment, the parameters were: $\mathrm{TE} / \mathrm{TR}=5 / 10 \mathrm{~ms}, \mathrm{FOV}=225 \mathrm{~mm}$, voxel size $=1.76 \times 1.76 \times 1.5 \mathrm{~mm}, \mathrm{FA}=40^{\circ}, \mathrm{NEX}=8$, number of slices $=60$, and duration for each scan $=10: 14 \mathrm{~min} / \mathrm{s}$. For the first bSSFP sequence, the RF excitation frequency, $f_{1}$, is determined by the MRI system itself. For the second bSSFP sequence, we have used an RF frequency, $f_{2}$, which is equal to $f_{1}+50 \mathrm{~Hz}$. Because TR is equal to $10 \mathrm{~ms}$, the frequency shift is chosen as $(2 \mathrm{TR})^{-1}=50 \mathrm{~Hz}$.
$\mathrm{B}_{0}$ map is the obtained by using the double echo method that involves 2 gradient echo images with different TEs. ${ }^{34}$ The sequence parameters in the phantom experiment were: $\mathrm{TE}=10$ or $15 \mathrm{~ms}, \mathrm{TR}=3500 \mathrm{~ms}, \mathrm{FOV}=225 \mathrm{~mm}$, voxel size $=1.76 \times 1.76 \times 1.5 \mathrm{~mm}, \mathrm{FA}=60^{\circ}$, number of slices $=20$, and duration for each scan $=7 / 33 \mathrm{~min} / \mathrm{s}$. In the human experiment the parameters were: $\mathrm{TE}=10$ or $15 \mathrm{~ms}, \mathrm{TR}=3500 \mathrm{~ms}, \mathrm{FOV}=225 \mathrm{~mm}$, voxel size $=$ $1.76 \times 1.76 \times 1.5 \mathrm{~mm}, \mathrm{FA}=60^{\circ}$, number of slices $=60$, and duration for each scan $=7 / 33 \mathrm{~min} / \mathrm{s}$. $\mathrm{T}_{2}$ map is obtained via a series of single echo spin-echo sequences with different TEs by using the exponential $\mathrm{T}_{2}$ fitting method whose result is accepted as the ground truth for many investigators. ${ }^{35,36}$ The sequence parameters in the phantom experiment were: $\mathrm{TE}=13,30,50,75,100$, or $150 \mathrm{~ms}$, $\mathrm{TR}=3500 \mathrm{~ms}$, FOV $=225 \mathrm{~mm}$, voxel size $=1.76 \times 1.76 \times 1.5 \mathrm{~mm}$, number of slices $=20$, and duration for each $\mathrm{scan}=7 / 33 \mathrm{~min} / \mathrm{s}$. In the human experiment, the parameters were: $\mathrm{TE}=13,40$, 75 , or $100 \mathrm{~ms}, \mathrm{TR}=6500 \mathrm{~ms}, \mathrm{FOV}=225 \mathrm{~mm}$, voxel size $=$ $1.76 \times 1.76 \times 1.5 \mathrm{~mm}$, number of slices $=60$, and duration for each scan $=14 / 00 \mathrm{~min} / \mathrm{s}$. Note that, the gradient echo and the spin echo sequences that we have mentioned above are all multi-slice sequences in which data are collected from all slices within $1 \mathrm{TR}$, and therefore the total duration of such multi-slice scans is dependent on TR and not dependent on the number of slices. Both of the $B_{0}$ and $T_{2}$ maps are obtained by using the RF excitation frequency equal to $f_{1}$. Experiments were conducted on $3 \mathrm{~T}$ Siemens Tim Trio MR scanner (Siemens, Erlangen, Germany) using a quadrature body coil.

For the human experiments, a Gaussian filter with a kernel size of $5 \times 5 \times 5$ voxels and a SD of 1.06 for each direction 

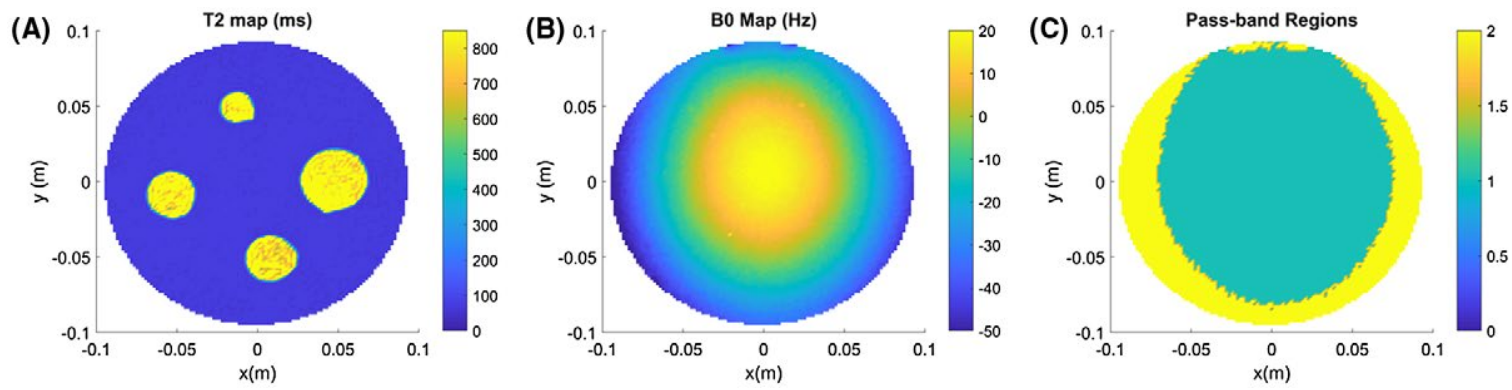

F I G URE 4 For the phantom experiment, (A) $\mathrm{T}_{2}$ map, (B) $\mathrm{B}_{0}$ map, and (C) the pass-band segments that are determined based on the $\mathrm{B}_{0}$ map of the phantom

was applied to the transceive phase data. No low-pass filter is applied to the data obtained in the phantom experiment.

\section{\begin{tabular}{l|l}
4 & RESULTS
\end{tabular}}

Magnitude and phase of the phantom images obtained by the 2 bSSFP sequences are shown in Figure 3A-D. Figure 3A and $\mathrm{C}$ correspond to the RF alternating bSSFP sequence applied by using the RF frequency that is determined by the MRI system, $f_{1}$. Figure $3 \mathrm{~B}$ and $\mathrm{D}$ correspond to the RF alternating bSSFP sequence applied using the shifted RF excitation frequency, $f_{2}=f_{1}+50 \mathrm{~Hz}$. Banding artifacts in the magnitude images, characterized by loss of signal, are shown by white arrows (Figure $3 \mathrm{~A}$ and $\mathrm{B}$ ). It is observed that, as expected, banding artifacts lie in the iso- $\mathrm{B}_{0}$ lines in both images (refer to the $\mathrm{B}_{0}$ map in Figure $4 \mathrm{~B}$ ). In the phase maps, as shown in Figure $3 \mathrm{C}$ and $\mathrm{D}$, a phase shift of $\pi$, radians is observed in the banding artifact region. This shift is not phasewrapping, because it occurs along several pixels as opposed to phase-wrapping that occurs in a single pixel transition by an amount of $2 \pi$.

$\mathrm{T}_{2}$ and $\mathrm{B}_{0}$ maps for the phantom are shown in Figure 4A and $B$. It is found that $T_{2}$ values are $70 \mathrm{~ms}$ and $850 \mathrm{~ms}$, on average, for the background and anomaly regions, respectively. $\mathrm{B}_{0}$ map demonstrates that there are deviations from central frequency, from $-70 \mathrm{~Hz}$ to $30 \mathrm{~Hz}$, indicating the extent of $\mathrm{B}_{0}$ inhomogeneity. The pass-band segments, which are determined by using the $\mathrm{B}_{0}$ map, are shown in Figure $4 \mathrm{C}$. Cyan region corresponds to pixels that are away from banding regions in the first image and the yellow region corresponds to pixels that are away from banding regions in the second image. Putting it differently, cyan region corresponds to pixels that fall in the pass-band segment of the first bSSFP experiment and yellow region corresponds to the pass-band segment of the second bSSFP experiment.

The phase of the collaged image, type-I corrected phase image, and type-II corrected phase image are shown in Figure $5 \mathrm{~A}-\mathrm{C}$. Note that the phase jumps (for an amount of $\pi$ ) that are observed in the collaged image are corrected in the type-I corrected phase image as expected. Moreover, phase errors because of finite $T_{2}$ values $\left(E_{2}<1\right.$, non-constant plateau effect) are also corrected for in the type-II corrected phase image. To appreciate the extent of the correction based on $\mathrm{T}_{2}$ values, Figure 5D shows the difference of type-II corrected phase image and type-I corrected phase image. The range of the difference image is approximately \pm 0.06 radians $\left( \pm 3.5^{\circ}\right)$. Phase differences between type-I and type-II corrections are largest near the band transition boundaries and also are discontinuous across them. In the error map (Figure 5D), it is found that the phase error is small in the anomalies, and this is because of the fact that they have large $T_{2}$ values (Figure 4A). Although the phase errors between type-I and type-II corrected images may be considered to be small for many applications, it will be shown below that use of type-II correction is essential, especially when conductivity reconstructions are considered.

Figure 5E displays the expected (theoretical) phase differences between type-I and type-II corrections as a function of $\mathrm{T}_{2}$ and $\Delta \mathrm{B}_{0}$ as calculated by using Equations (10) and (11). A phase difference does not occur if $\Delta \mathrm{B}_{0}$ is 0 or a multiple of 1/2TR (as also shown in Figure 2). It is also observed that phase differences between type-I and type-II corrections are largest near the boundaries between pass-band and stop-band regions and are also discontinuous across them. The reason why the error is largest near the boundaries between passband and stop-band regions is that at these boundaries, $\Delta \mathrm{B}_{0}$ corresponds to $\frac{1}{4 \mathrm{TR}}+\frac{n}{2 \mathrm{TR}}$ where $n$ is an integer, and these are the off-resonance frequencies at which $\mathrm{T}_{2}$ has maximal effect. Discontinuity of the error occurs because of the fact that $\mathrm{T}_{2}$ contribution to phase error, $\angle(M)$, changes sign across the boundary. With similar reasoning, discontinuity of the error in Figure 5D is because of the fact that pixels on either sides of the phase transition boundary are taken from different bSSFP images, and therefore $\mathrm{T}_{2}$ contributions to phase error, $\angle(M)$, has opposite polarities across the boundary.

Conductivity maps that are obtained from uncorrected (original) bSSFP phase images using the phase-based crMREPT technique are shown in Figure 6A and B. Severe conductivity artifacts occur in the banding artifact regions 

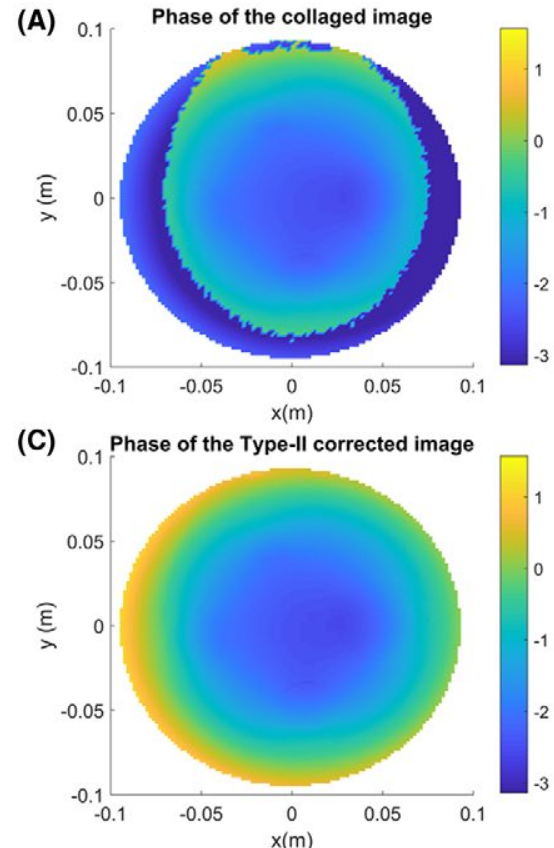

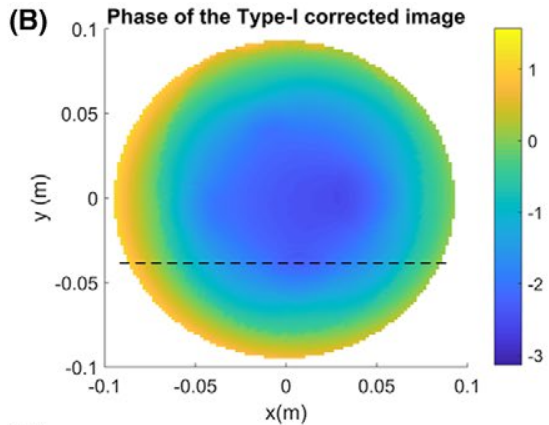

(D)

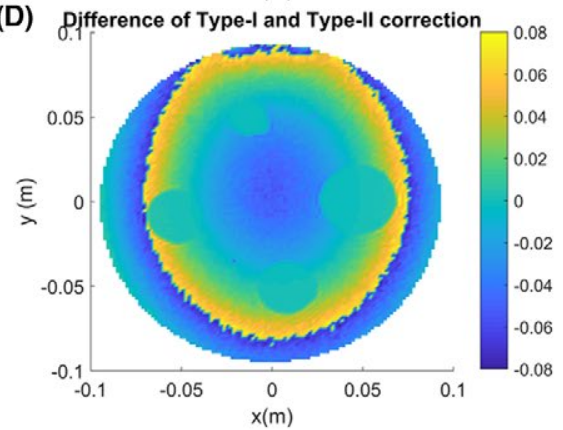

(E)

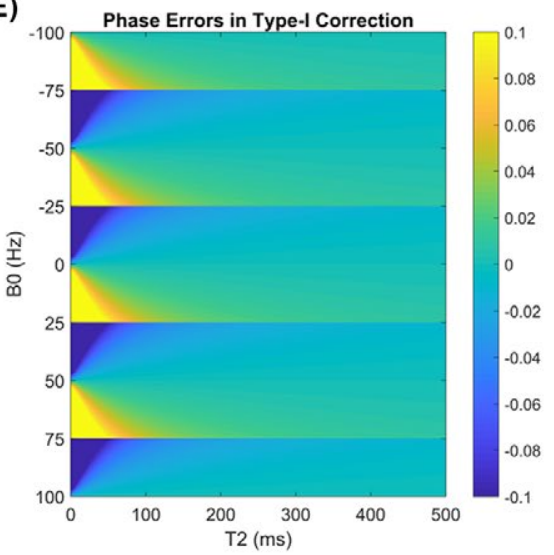

F I G URE 5 For the phantom experiment, (A) phase of the collaged image, (B) phase of the type-I corrected image, (C) phase of the type-II corrected image, (D) difference between type-I and type-II corrected phase images, and (E) theoretical difference between type-I and type-II corrected phases as a function of $\Delta \mathrm{B}_{0}$ and $\mathrm{T}_{2}$. The dotted line in (B) indicates the position of the profile plots shown in Figure 7. The unit of phase in all images is radians

F I G URE 6 For the phantom experiment, (A) conductivity map obtained from the phase image of the first bSSFP, (B) conductivity map obtained from the phase image of the second bSSFP, (C) conductivity map obtained from type-I corrected image, and (D) conductivity map obtained from type-II corrected image. The ring artifact shown in $(\mathrm{C})$ by an arrow is because of the phase ripple observed along the band transition boundary as shown in Figure 7
(A)
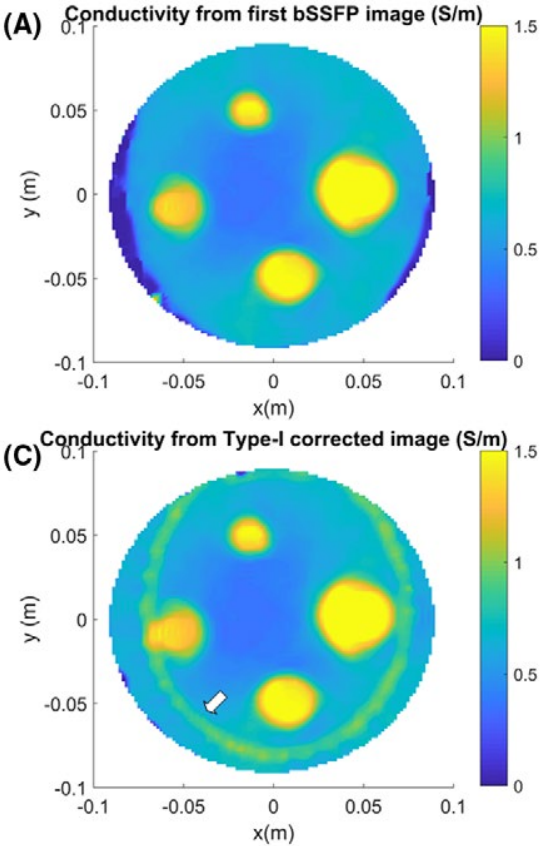

(B)

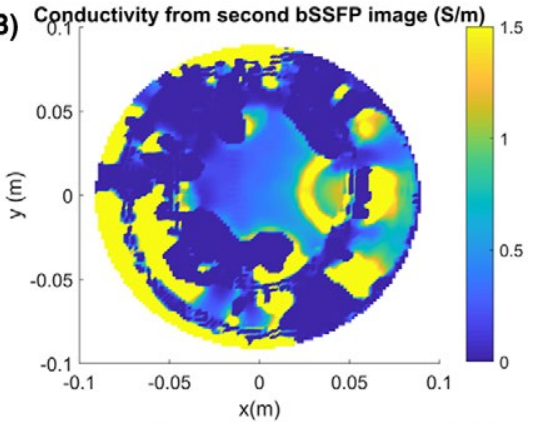

(D)

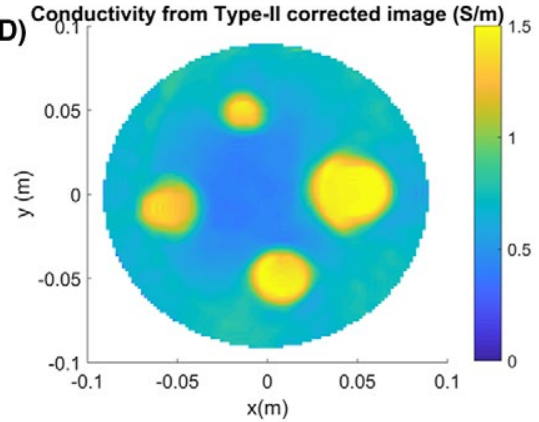

in both figures, even in regions where banding artifact is not fully onset. When the type-I corrected phase image is used for conductivity reconstruction, significant improvement in conductivity image is obtained as shown in Figure 6C. However, in this case, there is still distortion in the conductivity reconstruction along the band transition boundary. These are caused by the errors in type-I corrected images because of the fact that $\mathrm{T}_{2}$-based corrections are still not performed, which are most prominent along and near the band transition boundaries (the ring artifact shown in Figure 6C). Although phase errors in type-I are small, they still distort the conductivity image because their effects are amplified by Laplacian operation. Nonetheless, when the type-II corrected phase image is used to reconstruct the conductivity, 
F I G URE 7 For the phantom experiment, (A) phase of the type-I corrected image drawn as 3D surface plot, (B) phase of the type-II corrected image drawn as 3D surface plot, (C) phase profile of the type-I corrected image, (D) phase profile of the type-II corrected image, (E) conductivity profile of the type-I corrected image, and (F) conductivity profile of the type-II corrected image. The profiles are drawn along the line depicted in Figure $5 \mathrm{~B}$
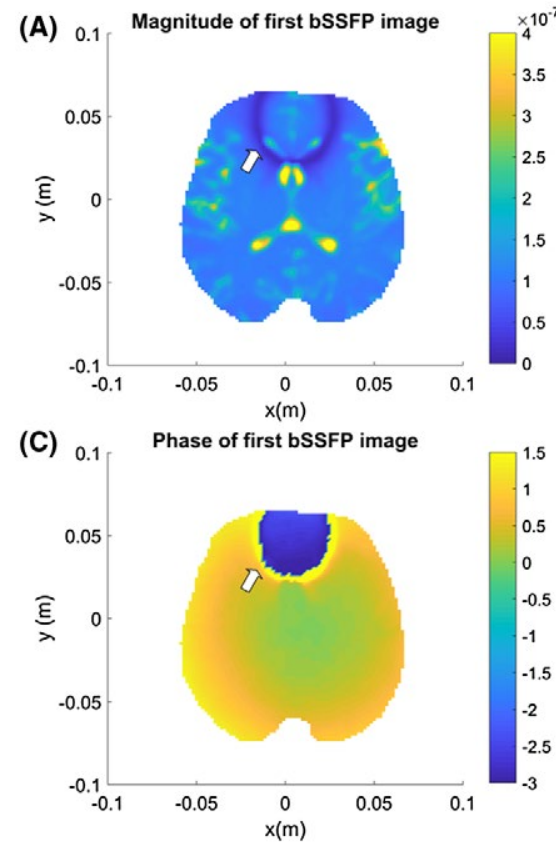

(A)
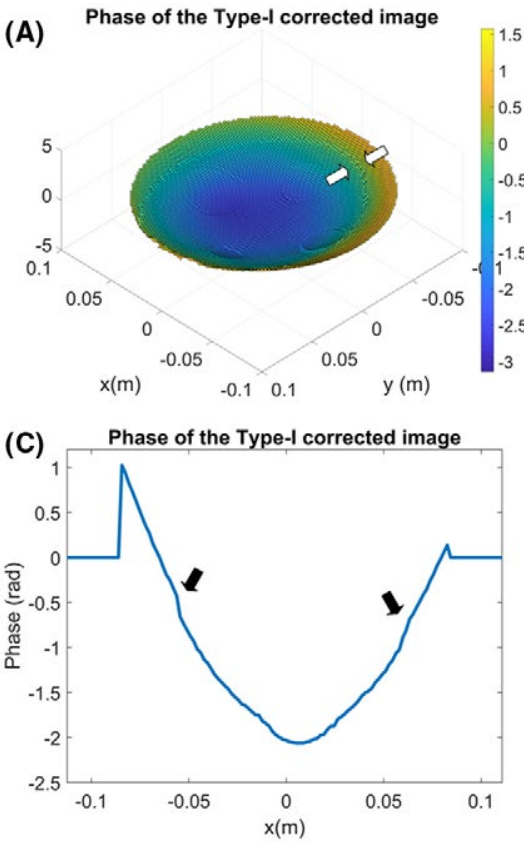

(E) Conductivity from the Type-I corrected image

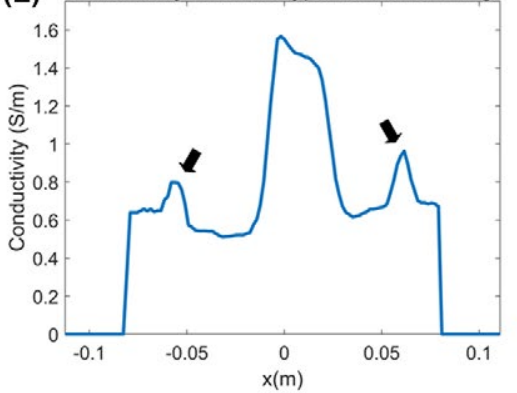

(B)
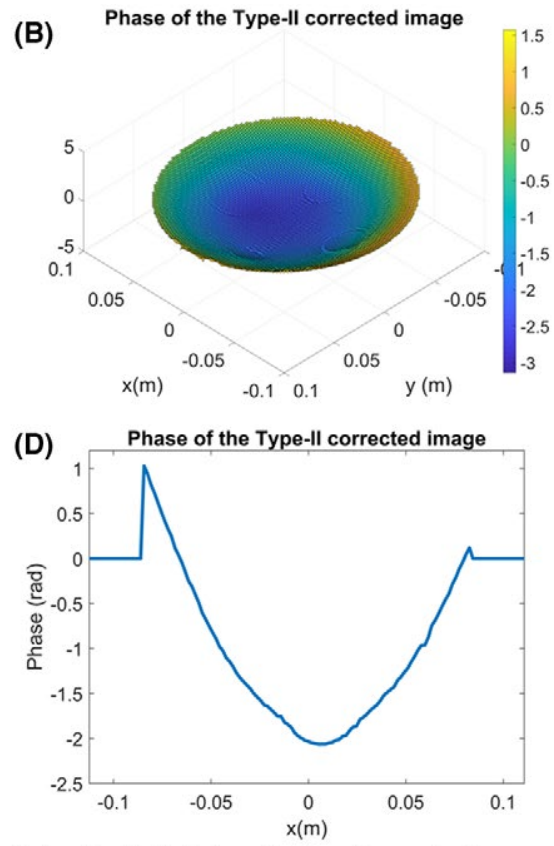

(F) Conductivity from the Type-ll corrected image

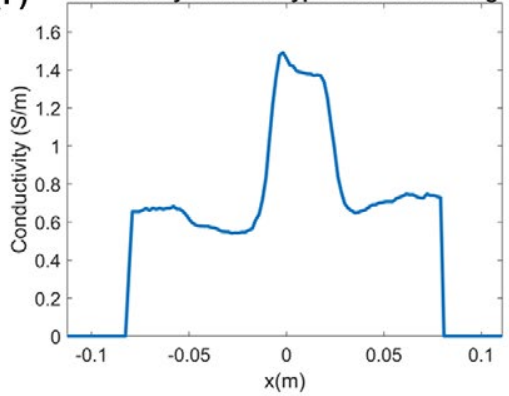

F I G URE 8 For the human experiment, (A) magnitude and (B) phase images of the first bSSFP sequence, (C) magnitude and (D) phase images of the second bSSFP sequence. Banding artifacts are shown by white arrows 

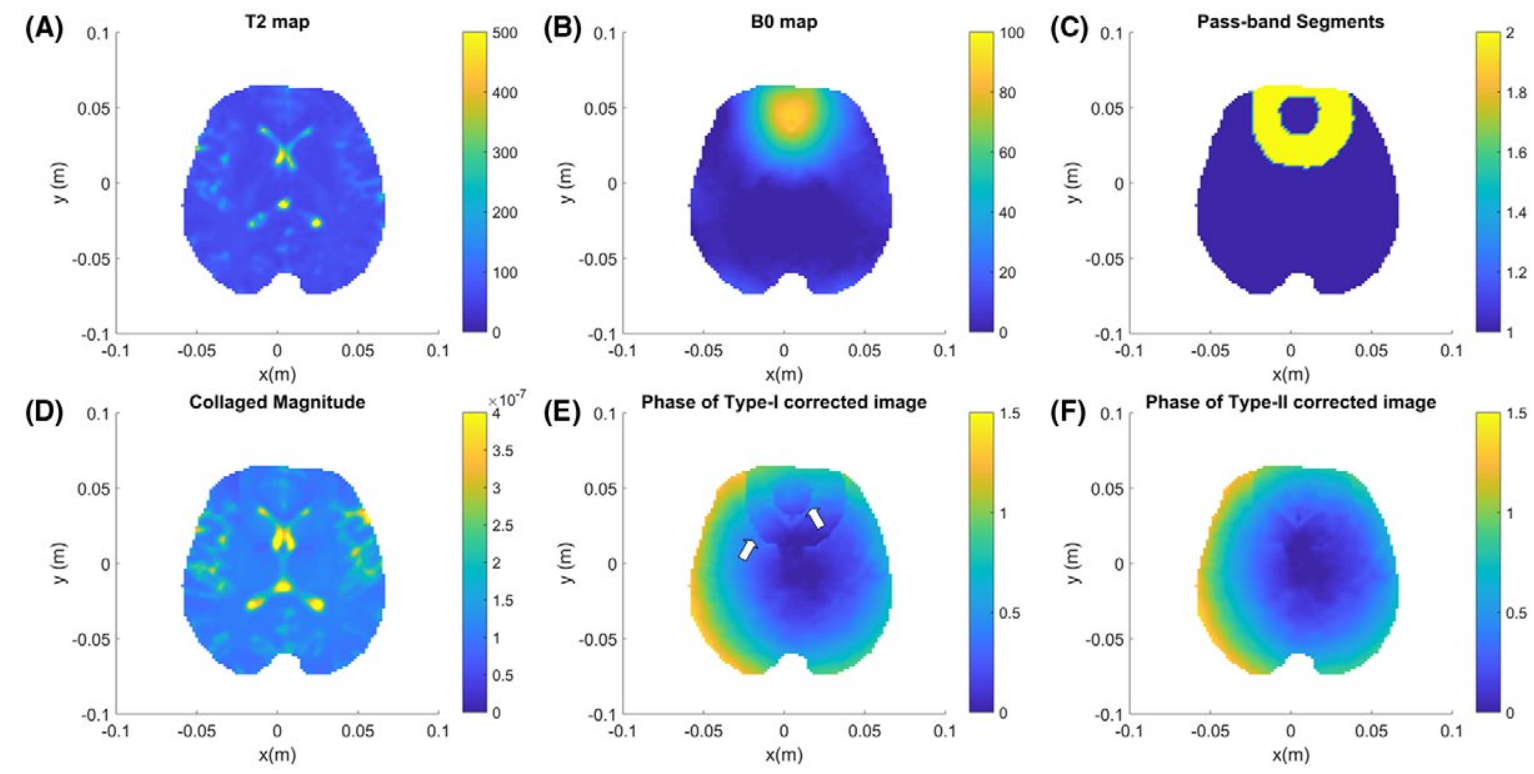

F I G U RE 9 For the human experiment, (A) $\mathrm{T}_{2}$ map, (B) $\mathrm{B}_{0}$ map, (C) the images of the pass-band segments that are determined based on the $\mathrm{B}_{0}$ map, (D) collaged magnitude, (E) phase of the type-I corrected image, and (F) phase of the type-II corrected image. Small phase ripples at the band transition boundary are shown by white arrows
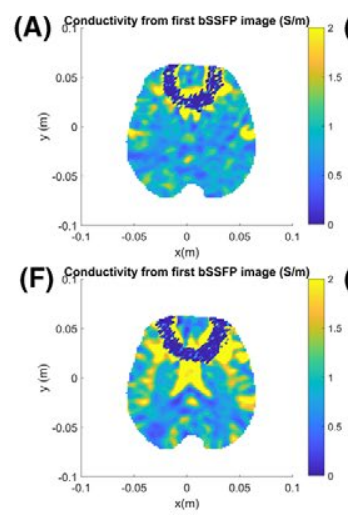

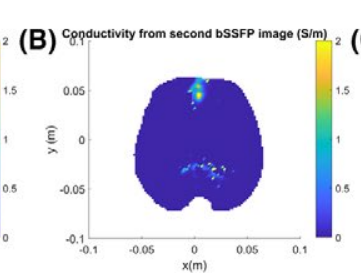

$(\mathbf{G})^{\mathrm{c}}$

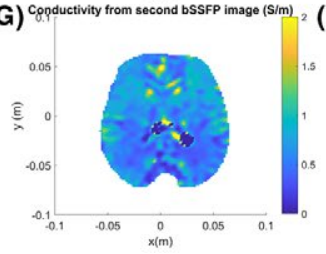

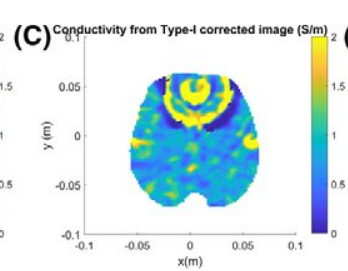

(H) $)^{\text {co }}$

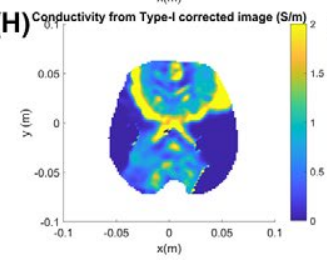

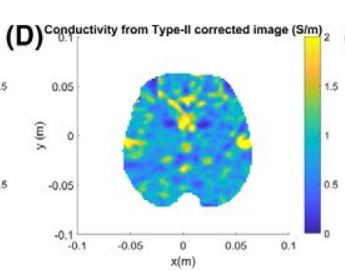

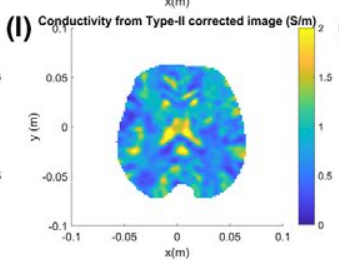

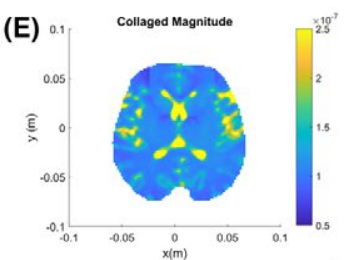

(J)

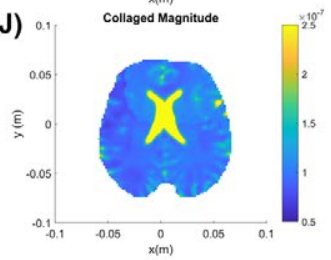

F I G URE 10 For the human experiment, reconstructed conductivities for 2 different slices of the brain for the human experiment. For the first slice, (A) conductivity map obtained from the first bSSFP phase image, (B) conductivity map obtained from the second bSSFP phase image, (C) conductivity map obtained from type-I corrected phase image, (D) conductivity map obtained from type-II corrected phase image, and (E) collaged magnitude image. (F-J) Corresponding images for the second slice. Although (A), (B), (F), and (G) suffer from banding artifact, (C) and (H) suffer from non-constant plateau effect, (D) and (I) are free from such disturbances

all artifacts are eliminated and a clean conductivity map is obtained (Figure 6D).

Figure 7A and B represent the type-I and type-II corrected phase images drawn as 3D surface plot. Small phase deviations near the band transition boundaries in type-I corrected phase images are visible in Figure 7A and are shown by white arrows. Along the line that is depicted in Figure 5B, profiles for phase (Figure 7C and D) and conductivity (Figure 7E and F) for both type-I and type-II corrections are shown. Both the small phase deviations and the conductivity ripples corresponding to the band transition boundaries are shown by black arrows (Figure 7C and E). Clearly, when type-II corrected phase images are used, ripples in the conductivity errors are significantly reduced.
Human experiment results are shown in Figures 8-10. Figure 8 demonstrates the magnitude and phase of both bSSFP runs for a middle axial slice. Banding artifacts are shown by white arrows. $\mathrm{T}_{2}$ map, $\mathrm{B}_{0}$ map and pass-band segments, for the same slice, are shown in Figure 9A-C. Collaged magnitude is shown in Figure 9D. Type-I and typeII corrected phase images are shown in Figure 9E and F. Although type-I corrected phase image suffers from the small deviations and jumps because of non-constant plateau effect (shown by white arrows), those errors are eliminated in the type-II corrected phase image. 3D version of phase-based crMREPT is used to obtain conductivity maps for 10 slices, and 2 different slices are shown in Figure 10. Because of banding artifact, there are severe distortions on conductivity maps 
obtained from both bSSFP runs (Figure 10A, B, F, and G) and the effect of the non-constant plateau is seen in Figure $10 \mathrm{C}$ and $\mathrm{H}$, but conductivity images that are obtained from type-II corrected phase (Figure 10D and I) do not include such distortions. Collaged bSSFP magnitudes for the 2 slices are also shown (Figure 10E and $\mathrm{J}$ ) for comparison with the reconstructed conductivity images.

\section{DISCUSSION AND CONCLUSIONS}

It has been argued that bSSFP is the most favorable phasemeasuring method for MREPT because of its speed, motion insensitivity, automatic eddy current compensation, and high SNR. ${ }^{14,29}$ However, bSSFP phase is susceptible to off-resonance and $\mathrm{T}_{2}$ effects, and it is vulnerable especially in certain regions where banding artifact occurs. In this study, we have addressed this problem and have developed a method for obtaining correct RF phase throughout the object. It has also been shown that the correction method provides phase distributions that have sufficient quality for obtaining conductivity images.

We have demonstrated the use of our proposed bSSFP phase correction method in experiments performed in a $3 \mathrm{~T}$ scanner. The method proposed in this work is not dependent on the static magnetic field strength, but because, to our knowledge, high field systems have higher $\mathrm{B}_{0}$ inhomogeneity, the use of the proposed method is even more indicated for high field systems.

The method that we have developed requires the acquisition of $\mathrm{T}_{2}$ and $\mathrm{B}_{0}$ maps as well as 2 bSSFP runs. In this article, the $\mathrm{T}_{2}$ and $\mathrm{B}_{0}$ mapping techniques that we have used take $45 \mathrm{~min}$ and $15 \mathrm{~min}$, respectively, for the phantom experiment. In this study, we have preferred to use a reliable $\mathrm{T}_{2}$ mapping technique that is considered to be a golden truth by many investigators, ${ }^{35,36}$ although there are faster methods that may provide considerable reduction in the acquisition time. For example, a method based on multispin-echo sequence is proposed in Ben-Eliezer et al., ${ }^{35}$ and an involved compensation technique for the interfering stimulated echoes is used. Another method ${ }^{36}$ using 1 single scan based on a triple echo steady-state (TESS) sequence makes use of modeling the relation between the echoes and an iterative numerical solution for obtaining the $T_{2}$ map. MIRACLE $^{37}$ is similar to TESS and merges TESS with a balanced acquisition scheme for motion-insensitive rapid configuration relaxometry. All these methods can be used to decrease $\mathrm{T}_{2}$ mapping time to 5-10 min. However, they need to be carefully optimized for SNR and artifacts so that they are acceptable for MREPT. Other approaches that are based on estimating $\mathrm{T}_{1}, \mathrm{~T}_{2}, \Delta \mathrm{B}_{0}$, and potentially also RF phase simultaneously from a model of the bSSFP data, such as LORE ${ }^{31}$ and PLANET, ${ }^{38}$ can also be implemented for obtaining the RF phase directly and within 5-10 min. Although these methods are implemented in Björk et al. ${ }^{31}$ and Shcherbakova et al., ${ }^{38}$ results for RF phase are not explicitly presented. Therefore, these methods need to be assessed for their performance with respect to RF phase and its use in MREPT. By introducing the aforementioned methods, scanning time might be reduced significantly, and therefore bSSFP may still maintain its comparative advantages for obtaining conductivity images.

Conductivity reconstructions obtained for the experimental phantom have well-defined internal boundaries and reasonably accurate conductivity values. Conductivity reconstructions of the in vivo human brain correlate with the bSSFP magnitude images that roughly correspond to the anatomical structures. However, there are several approaches by which they can be improved. As for any multi-scan-based method, our proposed method would also be affected from the patient motion between scans. Although algorithms can be devised for correcting motion errors, we think one should be cautious in using them because they may result in jumps and distortions in conductivity reconstructions because of the Laplacian operation involved. Rather, by taking precautions against motion and by using faster data acquisition techniques, which are discussed above, conductivity errors caused by motion might be reduced. There may be phase offset between scans, for example, if the excitation frequency for each scan is not set accurately. We have paid attention to scanner adjustments, and we have not encountered phase offsets while working with Siemens $3 \mathrm{~T}$ Tim Trio scanner. In addition, several pre- and post-processing techniques may also be used for further improvement: better estimation of the Laplacian of the phase, ${ }^{28}$ use of proper combination of multi-channel data, ${ }^{39}$ using adaptive regularization, ${ }^{40}$ and increasing the number of acquisitions for signal averaging are some of the possibilities. In our experiments, to obtain banding artifact with even smaller $\Delta \mathrm{B}_{0}$ values, and therefore be able to demonstrate the effectiveness of our algorithms, high values for TE/TR pair are used $(5 / 10 \mathrm{~ms})$. Using lower $\mathrm{TE}$ values (e.g. $\mathrm{TE} / \mathrm{TR}=2.5 / 5 \mathrm{~ms}$ ) would increase $\mathrm{SNR}$ and would also reduce the acquisition time. As mentioned before, using phase-based formulation rather than complex $\mathrm{B}_{1}^{+}$-based formulation generates additional bias in the whole conductivity image, especially toward the boundary of the imaged object. ${ }^{24}$ Therefore, the phase correction method proposed in this study may also be used in complex $\mathrm{B}_{1}^{+}$-based MREPT techniques.

In this article, we have used different excitation frequencies-the difference being equal to $(2 \mathrm{TR})^{-1}$ - for the 2 bSSFP sequence applications. However, instead of shifting the excitation frequency, one can also use RF phase cycling. In such a case, the formula for the complex image value, at an arbitrary pixel in the slice of interest becomes: 


$$
S=K M=K M_{1} e^{-T E / T_{2}} e^{i 2 \pi f_{O R} T E} \frac{1-\mathrm{ae}^{-i\left(2 \pi f_{O R} T R+\phi_{n}\right)}}{1-b \cos \left(2 \pi f_{O R} T R+\phi_{n}\right)}
$$

where $\phi_{n}$ is the phase increment of RF pulse in each TR. When $\phi_{n}$ equals to $180^{\circ}+\Delta f_{s}$, this formula leads to the formula for the alternating RF pulse with the excitation frequency shift of $\Delta f_{s}$ case (the formula used in this study) with a small difference: magnitude-wise, frequency shift, and RF phase cycling yield the same result; however, there is an additional factor of $e^{i 2 \pi \Delta f_{s} T E}$ that affects the phase. With the selection of $\Delta f_{s}=(2 \mathrm{TR})^{-1}=(4 \mathrm{TE})^{-1}$, phase difference between frequency shift and RF phase cycling becomes $\pi / 2$. The method for phase correction can be easily modified to compensate for this $\pi / 2$ offset.

It has been argued in the Methods section that by making use of methods for determining the off-resonance and $\mathrm{T}_{2}$ values for each pixel, one can obtain the correct value of transceive phase for each pixel using Equations (10) and (11). This would even be possible with only $1 \mathrm{bSSFP}$ run therefore avoiding the additional scan time of the second run. However, there is a fundamental drawback of Equations (10) and (11) for $f_{\mathrm{OR}}$ values close to the boundaries between pass-band and stop-band regions. In these boundary regions, $f_{\mathrm{OR}}$ dependence of phase is very steep, and therefore even a small error in the measured $f_{\mathrm{OR}}$ value would result in an overly erroneous phase value. To avoid these ill-conditioned regions of the magnetization versus off-resonance graphs, we have chosen to use 2 bSSFP runs and use the pass-band regions of either of them.

\section{REFERENCES}

1. Barber C, Brown B, Freeston I. Imaging spatial distributions of resistivity using applied potential tomography. Electron Lett. 1983;19:933.

2. McEwan A, Cusick G, Holder D. A review of errors in multi-frequency EIT instrumentation. Physiol Meas. 2007;28:S197-S215.

3. Griffiths H. Magnetic induction tomography. Meas Sci Technol. 2001;12:1126-1131.

4. Woo EJ, Lee SY, Mun CW. Impedance tomography using internal current density distribution measured by nuclear magnetic resonance. In: Proceedings of SPIE 2299, Mathematical Methods in Medical Imaging III; 1994; San Diego, CA, pp. 377-385.

5. Ider YZ, Birgul O. Use of the magnetic field generated by the internal distribution of injected currents for electrical impedance tomography (MR-EIT). Elektrik. 1998;6:215-225.

6. Seo JK, Yoon JR, Woo EJ, Kwon O. Reconstruction of conductivity and current density images using only one component of magnetic field measurements. IEEE Trans Biomed Eng. 2003;97:1121-1124.

7. Ider YZ, Onart S. Algebraic reconstruction for $3 \mathrm{D}$ magnetic resonance-electrical impedance tomography (MREIT) using one component of magnetic flux density. Physiol Meas. 2004;25:281-294.
8. Seo JK, Kwon O, Woo EJ. Magnetic resonance electrical impedance tomography (MREIT): conductivity and current density imaging. J Phys Conf Ser. 2005;12:140-155.

9. Ozparlak L, Ider Y. Induced current magnetic resonance-electrical impedance tomography. Physiol Meas. 2005;26:S289-S305.

10. Haacke E, Petropoulos L, Nilges E, Wu D. Extraction of conductivity and permittivity using magnetic resonance imaging. Phys Med Biol. 1991;36:723-734.

11. Wen H. Noninvasive quantitative mapping of conductivity and dielectric distributions using RF wave propagation effects in high-field MRI. In: Proceedings of SPIE 5030, Medical Imaging: Physics of Medical Imaging; 2003; San Diego, CA.

12. Katscher U, Voigt T, Findeklee C, Vernickel P, Nehrke K, Dossel O. Determination of electric conductivity and local SAR Via B1 mapping. IEEE Trans Med Imaging. 2009;28:1365-1374.

13. Liu J, Wang Y, Katscher U, He B. Electrical properties tomography based on B1 maps in MRI: principles, applications, and challenges. IEEE Trans Biomed Eng. 2017;64:2515-2530.

14. Katscher U, van den Berg C. Electric properties tomography: biochemical, physical and technical background, evaluation and clinical applications. NMR Biomed. 2017;30:e3729.

15. Hafalir F, Oran O, Gurler N, Ider Y. Convection-reaction equation based magnetic resonance electrical properties tomography (crMREPT). IEEE Trans Med Imaging. 2014;33:777-793.

16. Liu J, Zhang X, Schmitter S, Van de Moortele P, He B. Gradientbased electrical properties tomography (gEPT): a robust method for mapping electrical properties of biological tissues in vivo using magnetic resonance imaging. Magn Reson Med. 2014;74:634-646.

17. Balidemaj E, van den Berg C, Trinks J, et al. CSI-EPT: a contrast source inversion approach for improved MRI-based electric properties tomography. IEEE Trans Med Imaging. 2015;34:1788-1796.

18. Stollberger R, Wach P. Imaging of the active B1 field in vivo. Magn Reson Med. 1996;35:246-251.

19. Yarnykh V. Actual flip-angle imaging in the pulsed steady state: a method for rapid three-dimensional mapping of the transmitted radiofrequency field. Magn Reson Med. 2006;57:192-200.

20. Sacolick L, Wiesinger F, Hancu I, Vogel M. B1 mapping by Bloch-Siegert shift. Magn Reson Med. 2010;63:1315-1322.

21. Katscher U, Kim D, Seo J. Recent progress and future challenges in MR electric properties tomography. Comput Math Methods Med. 2013;2013:1-11.

22. van Lier A, Brunner D, Pruessmann K, et al. B1+ Phase mapping at $7 \mathrm{~T}$ and its application for in vivo electrical conductivity mapping. Magn Reson Med. 2011;67:552-561.

23. Voigt T, Katscher U, Doessel O. Quantitative conductivity and permittivity imaging of the human brain using electric properties tomography. Magn Reson Med. 2011;66:456-466.

24. van Lier A, Raaijmakers A, Voigt T, et al. Electrical properties tomography in the human brain at 1.5, 3, and 7T: a comparison study. Magn Reson Med. 2013;71:354-363.

25. Gurler N, Ider Y. Gradient-based electrical conductivity imaging using MR phase. Magn Reson Med. 2017;77:137-150.

26. Kim D, Choi N, Gho S, Shin J, Liu C. Simultaneous imaging of in vivo conductivity and susceptibility. Magn Reson Med. 2013;71:1144-1150.

27. Gho S, Shin J, Kim M, Kim D. Simultaneous quantitative mapping of conductivity and susceptibility using a double-echo 
ultrashort echo time sequence: example using a hematoma evolution study. Magn Reson Med. 2015;76:214-221.

28. Lee S, Bulumulla S, Wiesinger F, Sacolick L, Sun W, Hancu I. Tissue electrical property mapping from zero echo-time magnetic resonance imaging. IEEE Trans Med Imaging. 2015;34:541-550.

29. Stehning C, Voigt TR. Katscher U. Real-time conductivity mapping using balanced SSFP and phase-based reconstruction. In: Proceedings of the 19th Annual Meeting of ISMRM; 2011; Montreal, Canada. p. 128.

30. Lauzon M, Frayne R. Analytical characterization of RF phase-cycled balanced steady-state free precession. Concepts Magn Reson A. 2009;34A:133-143.

31. Björk M, Ingle R, Gudmundson E, Stoica P, Nishimura D, Barral J. Parameter estimation approach to banding artifact reduction in balanced steady-state free precession. Magn Reson Med. 2013;72:880-892.

32. Scheffler K, Heid O, Hennig J. Magnetization preparation during the steady state: fat-saturated 3D TrueFISP. Magn Reson Med. 2001;45:1075-1080.

33. Li C, Yu W, Huang S. An MR-based viscosity-type regularization method for electrical property tomography. Tomography. 2017;3:50-59.

34. Jezzard P, Balaban R. Correction for geometric distortion in echo planar images from B0 field variations. Magn Reson Med. 1995;34:65-73.

35. Ben-Eliezer N, Sodickson D, Block K. Rapid and accurate T2 mapping from multi-spin-echo data using Bloch-simulationbased reconstruction. Magn Reson Med. 2014;73:809-817.

36. Heule R, Ganter C, Bieri O. Triple echo steady-state (TESS) relaxometry. Magn Reson Med. 2013;71:230-237.

37. Nguyen D, Bieri O. Motion-insensitive rapid configuration relaxometry. Magn Reson Med. 2017;78:518-526.

38. Shcherbakova Y, van den Berg C, Moonen C, Bartels L. PLANET: an ellipse fitting approach for simultaneous T1 and T2 mapping using phase-cycled balanced steady-state free precession. Magn Reson Med. 2018;79:711-722.

39. Lee J, Shin J, Kim D. MR-based conductivity imaging using multiple receiver coils. Magn Reson Med. 2016;76:530-539.

40. Ropella K, Noll D. A regularized, model-based approach to phase-based conductivity mapping using MRI. Magn Reson Med. 2017;78:2011-2021.

\section{APPENDIX 1}

Phase error for the special case of $\mathbf{E}_{2}=1$

Phase error, $\angle(M)$, is investigated for a specific case where $\mathrm{E}_{2}=1$. To make Equation (11) more tractable, new definitions are made:

$$
\angle(M)=\phi^{\prime}+\theta^{\prime}
$$

where,

$$
\begin{array}{r}
\phi^{\prime}=2 \pi\left(\mathrm{f}_{\mathrm{OR}}+\Delta \mathrm{f}_{s}\right) \mathrm{TE}, 2 \phi^{\prime}=2 \pi\left(\mathrm{f}_{\mathrm{OR}}+\Delta \mathrm{f}_{s}\right) \mathrm{TR}, \\
\text { and } \theta^{\prime}=\angle\left(1+1 e^{-i 2 \phi^{\prime}}\right) .
\end{array}
$$

With these definitions:

$\tan \left(\theta^{\prime}\right)=\frac{\sin \left(-2 \phi^{\prime}\right)}{1+\cos \left(-2 \phi^{\prime}\right)}=\frac{2 \sin \left(-\phi^{\prime}\right) \cos \left(-\phi^{\prime}\right)}{2 \cos ^{2}\left(-\phi^{\prime}\right)}=\tan \left(-\phi^{\prime}\right)$.

Because the tangent function is periodic with $\pi$, relationship between $\theta^{\prime}$ and $\phi^{\prime}$ becomes:

$$
\theta^{\prime}=k \pi-\phi^{\prime},
$$

where $k$ is any integer. Therefore,

$$
\angle(M)=\phi^{\prime}+k \pi-\phi^{\prime}=k \pi .
$$

We conclude that for the pixels that have infinite $\mathrm{T}_{2}$ value, $\angle(M)$ is effectively either 0 or $\pi$.

How to cite this article: Ozdemir S, Ider YZ. bSSFP phase correction and its use in magnetic resonance electrical properties tomography. Magn Reson Med. 2019;81:934-946. https://doi.org/10.1002/mrm.27446 